\title{
Politische Träume in der römischen Kaiserzeit. Deuter, Deutung und Verbreitung
}

\author{
Gregor Weber
}

\section{Einführung}

Der Historiker Cassius Dio - geboren um 163 in Bithynien, gestorben nach 229 n. Chr. ${ }^{1}$ - berichtet in seiner „Römischen Geschichte“ ausführlich über seine eigene Zeit, vor allem über die Kaiser Commodus (180-192 n. Chr.) und Septimius Severus (193-211 n. Chr.). Dass Letzterer nach den Wirren des Fünfkaiserjahrs den Prinzipat errang, war Dio zufolge das Ergebnis „äußerst heftiger Kriege und inneren Auseinandersetzungen ". ${ }^{2}$ Dio formuliert, wie er dazu kam, über diese Ereignisse zu schreiben:

Ich hatte eine kleine Schrift über die Träume und Vorzeichen verfasst und herausgegeben, die Severus auf die Erlangung der Kaiserwürde hoffen ließen. Als er das von mir übersandte Exemplar gelesen hatte, antwortete er mir in einem ausführlichen und anerkennenden Schreiben. ${ }^{3}$

In der Nacht nach dem Erhalt des Antwortbriefes träumte Dio selbst, dass ihn

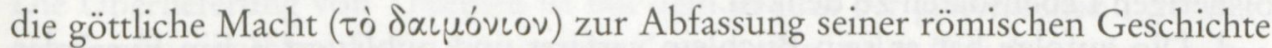
aufforderte, womit - wie so oft - eine göttliche Autorisierung in Anspruch genommen wurde. ${ }^{4}$ Die Schrift über die Träume scheint also eine Art Erstlingswerk gewesen zu sein. Die zitierte Passage ist aufschlussreich, weil sich daran einige für unser Thema relevante Aspekte festmachen lassen:

a. Cassius Dio berichtet weder etwas über den Zeitpunkt der Abfassung bzw. Publikation seiner Schrift noch über deren Zustandekommen. Es ist deshalb nicht zwingend, von einer Abfassung erst nach der Proklamation des Severus am 9. April 193 auszugehen, sondern denkbar ist auch die Zeit nach dem Tod des Commodus am 31. Dezember 192, als, wie es heißt, Severus „auf die Erlangung der Kaiserwürde hoffte“ - was umso riskanter für den Autor gewesen wäre.

\footnotetext{
${ }^{1}$ Zu ihm zuletzt: Hose 2007; Sidebottom 2007, 74-78.

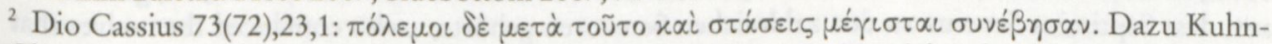
Chen 2002, 208. Zum historischen Kontext: Spielvogel 2006, 63-108; Lichtenberger 2011, $1 \mathrm{f}$.

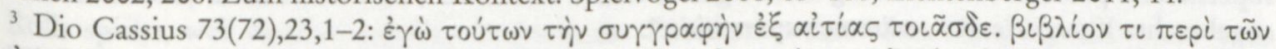

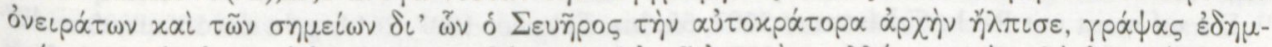

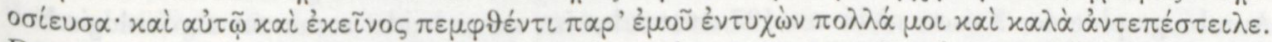
Dazu Birley 1988, 203 f.; Schmidt 1997, dort besonders 2613-2618; Freyburger-Galland 1999, 534 und 537; Weber 2000, 76-78; Sordi 2001, 393; Moscovich 2004, 358; Hose 2007, 462f.; Sidebottom 2007, 56 .

${ }^{4}$ Andere Werke auf Traumgeheiß sind z. B. Hesiods Erga, die Aitia des Kallimachos sowie die Oneirokritika des Artemidor.
} 
b. Es lässt sich nur vermuten, dass in dieser Schrift auch die im Traum vorkommenden Bilder, Worte und Symbole auf die Zukunft gedeutet wurden. Allerdings hat Dio den sechs in seiner Römischen Geschichte berichteten Träumen von Septimius Severus keine Deutung beigegeben und ging offenkundig davon aus, dass seine Leser sie verstanden und keiner weiteren Erläuterungen bedurften. ${ }^{5}$ Die Reaktion des Kaisers lässt sich aber in jedem Fall so interpretieren, dass ihn die Träume in seinen Ambitionen für die Zukunft bestärkten. ${ }^{6}$

c. Die Herkunft des verwendeten Traummaterials bleibt unklar: ${ }^{7}$ Es kann entweder von Dio stammen oder von anderen Personen, die sich unter Umständen Severus geneigt machen wollten, zumal die Zeitgenossen mit Interesse den Lebenslauf möglicher Prätendenten verfolgten. ${ }^{8}$ Vermutet wurden auch Träume des Kaisers selbst ${ }^{9}$ - doch sollte die Tätigkeit des Historikers allein darin bestanden haben, das ihm übermittelte kaiserliche Material in eine annehmbare Form zu bringen? Wir wissen jedenfalls, dass Septimius Severus viele Vorzeichen, darunter auch Träume, in seiner ,Autobiographie' aufzeichnete und in öffentlichen Weihebildern umsetzen ließ, was eine beträchtliche Aufmerksamkeit hervorgerufen haben dürfte; ${ }^{10}$ allerdings lässt sich nichts Genaues über den Zeitpunkt der Abfassung des Werkes aussagen. ${ }^{11}$ Dass sich Träume des Severus selbst bereits lange vor seinem Herrschaftsantritt in Umlauf befanden, erscheint letztlich aber kaum wahrscheinlich, sondern es ist am ehesten an Konstrukte aus seinen bisherigen Lebensdaten zu denken.

d. Dio zufolge hat er sein Büchlein verfasst und publiziert. ${ }^{12}$ Ein Exemplar sandte er dem Kaiser, während wir von weiteren Abschriften weder die Zahl noch den Verbreitungsgrad kennen. Man wird sicherlich von mancher Lesung im Freundeskreis ausgehen dürfen, ebenso von der Zusendung an senatorische Standesgenossen und Freunde des Autors. ${ }^{13}$ Ob Dios kleine Schrift oder deren

\footnotetext{
5 Weber 2000, 202-210. Möglicherweise hat auch Xiphlinos in seinem Cassius Dio-Exzerpt die Deutungen ausgelassen. Zu einigen dieser Träume liegt eine Parallelüberlieferung bei Herodian und in der Historia Augusta vor.

6 Vgl. dazu auch Spielvogel 2006, 68.

7 Zu Dios Quellen: Kuhn-Chen 2002, 135-142; Moscovich 2004.

8 Zur langen Karriere des Septimius Severus vor 193: Birley 1988, 47-88.

9 Vgl. Schmidt 1997, 2613 f.: "einzig er [sc. der Kaiser] kann die Quelle seiner Träume sein“.

${ }_{10}$ Herodian 2,9,3f., dazu Birley 1988, $167 \mathrm{f}$. und 203; Chausson 1995, $184 \mathrm{f}$. und 188; Weber 2000, 109 f.; Moscovich 2004, 357 f. mit Anm. 5; Pausch 2004, 321 f., Anm. 82. Birley 1988, 41, geht davon aus, dass Septimius Severus zu den zahlreichen Senatoren gehörte, "who convinced themselves that supernatural powers were showing them signs of future eminence“, verwendet dafür jedoch die unangebrachte Kategorie der „extreme superstition“.

${ }_{11}$ Darauf weist mit Recht Sidebottom 2007, 55, hin. Schmidt 1997, 2614, geht davon aus, dass die Autobiographie des Kaisers nicht vor 197 entstanden ist; sie „scheidet somit aus chronologischen Gründen als mögliche Quelle für Dios Pamphlet aus. " Chausson 1995 plädiert für eine hohe Datierung, das heißt in die Jahre 197/98 n. Chr., anders Sordi 2001 mit einem Datum nach 202.

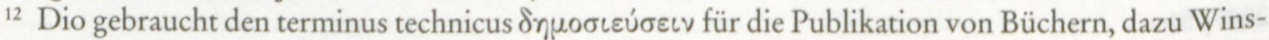
bury 2009, 86-91.

${ }_{13} \mathrm{Zu}$ den Stationen von der Abfassung bis zur Publikation, unter denen die recitatio eine wichtige
} 
Inhalt auch unter den in Pannonien stationierten Legionen, die die Kaiserausrufung vollzogen hatten, und den führenden Militärs bekannt wurde und hierbei eine - entscheidende? - Rolle gespielt hat, zumal Septimius Severus militärisch nicht gerade begabt war, ${ }^{14}$ lässt sich nicht sagen. ${ }^{15}$

Aus diesen Punkten wird deutlich, dass für zentrale Aspekte erhebliche Informationsdefizite vorliegen; sie führen jedoch zu weiteren Fragen, die im Folgenden abgehandelt werden: Zum einen gilt es zu klären, ob mit Septimius Severus und Cassius Dio ein Sonderfall vorliegt und wie es sich mit anderen Kaisern und anderen Historikern oder Biographen verhalten hat; zum anderen hat man zu fragen, in welchen politischen Kontexten Träume zu finden waren und wie mit ihnen, etwa im Hinblick auf ihre Publikation oder deren Unterdrückung, umgegangen wurde. Schließlich ist in den Blick zu nehmen, wer kaiserliche Träume gedeutet hat und auf welcher Wissensbasis dies geschah bzw. was ,Wissen' hier konkret meint. Zunächst möchte ich kurz darauf eingehen, warum Träume in der Antike als relevant erachtet wurden und was für unseren Kontext unter Divination und Zukunft zu verstehen ist.

\section{Die Relevanz von Träumen in der Antike}

Die Überlieferung von Träumen ist mit dem Beginn der griechischen Literatur, mit den homerischen Epen, verbunden: Zeus sandte in der Ilias dem griechischen Oberfeldherrn Agamemnon einen Traum, in dem der greise Nestor als Traumfigur agierte und zum erneuten Kampf gegen die Troianer aufforderte; die Umsetzung dieser direkten Traumbotschaft führte die Griechen - so war es Zeus' Plan - statt zur Eroberung Troias ins Verderben. Außerdem träumte Penelope in der Odyssee verschlüsselt von der Heimkehr des Odysseus und erzählte den Traum ihrem verkleidet heimgekehrten Mann, der ihn ihr deutete. ${ }^{16}$ Diese beiden Traumerzählungen dürften jedem Kind, das wie üblich mit Homer Lesen und Schreiben lernte, bekannt gewesen sein. Aus ihnen geht hervor, dass es Träume gab, die nicht gedeutet werden mussten, und solche, die der Deutung bedurften, wofür schon bei Homer Spezialisten - also Traumdeuter - genannt werden. ${ }^{17}$

Träume sind in allen literarischen Gattungen aus der Antike zu finden, ebenso in Papyri und Inschriften, ${ }^{18}$ und dass philosophische und medizinische Über-

Rolle spielte, weil sie eine wesentliche Form der Publikation darstellte: Winsbury 2009, 101-110; White 2009, 282 f. mit Anm. 282. Grundlegend ist Starr 1987, bes. 213-215.

${ }_{14}$ Zum beteiligten Personenkreis: Handy 2009, 101-105 und 115-128.

${ }^{15}$ Für andere Träume geht Birley 1988, 108 f., von einer aktiven Verbreitung durch „officers and agents" aus.

${ }_{16}$ Dazu Walde 2001b, 19-42; Weber 2003b, $13 \mathrm{f}$.

17 Homer, Ilias 1,62 f. u. 5,148ff., dazu Weber 2000, 44 mit Anm. 112, dort weitere Literatur.

${ }^{18} \mathrm{Vgl}$. Weber 2005-2006; Renberg 2010. 
legungen Träumen den übernatürlichen Ursprung absprachen, führte nicht dazu, dass Träume als bedeutungslos erachtet wurden. Denn in der Medizin konnten Träume zur Prognostik für Krankheiten verwendet werden, ${ }^{19}$ und außerdem gab es Träume, die - wie wir sagen würden - als Tagesreste angesehen wurden und dem Träumenden etwas über die eigene Befindlichkeit sagen konnten. Der entscheidende Unterschied zum modernen Traumverständnis besteht darin, dass es in der Antike nicht darum ging, über die Traumarbeit Einsichten in das eigene Ich oder in Prägungen durch die Vergangenheit zu erlangen, sondern Träume waren potentiell Fingerzeige für die Zukunft, sie vermittelten ein Wissen über die Zukunft bzw. konnten letztere auch beeinflussen.

Wie hingen nun diese Dinge nach antiker, vor allem stoischer Vorstellung zusammen? Indem Träume als Botschaften der Götter verstanden wurden, weisen sie in aller Regel einen klaren Zukunftsbezug auf, wie es im übrigen auch bei anderen Divinationsformen der Fall war. ${ }^{20}$ Weiter führend ist hier die Definition, die Fritz Graf gegeben hat:

Dabei geht es nicht nur um Zukunftswissen, sondern ebenso um die Kenntnis verborgener Gründe für bereits eingetretenes bedrohliches Geschehen. Ziel des divinatorischen Rituals ist es, mit dem Göttlichen in Kontakt zu treten, um Einsicht zu erhalten in dieses Wissen. ${ }^{21}$

Zwar war etlichen antiken Autoren zufolge das menschliche Schicksal, das fatum, unabänderlich. ${ }^{22}$ Dennoch wurde der Versuch als lohnend angesehen, sich in Entscheidungssituationen des Wissens über die Zukunft zu vergewissern, wofür eine große Palette an durchaus konkurrierenden Divinationsformen zur Verfügung stand. ${ }^{23}$ Denn auf diese Weise konnten Unsicherheiten und Ängste abgebaut werden, war „zukunftsgerichtetes Handeln möglich“: Das individuelle oder kollektive, weil den Staat betreffende Geschehen wurde durch die Divination „in eine verstehbare Kausalität eingebunden und damit beeinflußbar.“24

Träumen konnte prinzipiell jeder, vom Kaiser bis zum Sklaven, Männer und Frauen, und es wurde eher als bemerkenswert angesehen, wenn jemand wie Kai-

19 Holowchak 2001; Liatsi 2002; Gil 2004.

${ }^{20}$ Vgl. dazu die Beiträge und das Spektrum in Brodersen 2001; eine Übersicht auch bei Graf 1999.

${ }^{21}$ Graf 1999, 883, der - mit Beispielen belegt (884-886) - zwei Grundtypen der Divination unterscheidet, nämlich auf direktem Wege (Traum, Ekstase) oder mit Hilfe eines Mediums (Orakel).

${ }^{22}$ Zum „Verhältnis zwischen Göttern (bzw. numen), menschlichem Mitwirken und Schicksal im Rahmen der römischen Religion", vornehmlich in der Stoa: Frede 2001, 157 f.; Kuhlmann 2008, 174.

${ }^{23}$ Frede 2001, 157 f. Selbst Artemidor (1,6,15,19-23; 4,2,246,2-247,10) gesteht zu, dass von den Göttern geforderte Träume signifikant sein können, er grenzt sie aber strikt von magischen Praktiken ab (4,2,246,7-10): „Denke daran, wenn du Traumgesichte forderst, opfere weder Weihrauch noch sprich Zauberformeln und, um die Hauptsache zu sagen, frage die Götter nichts Nebensächliches“

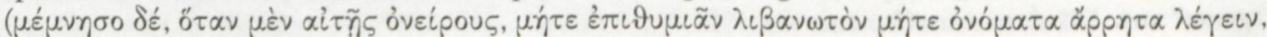

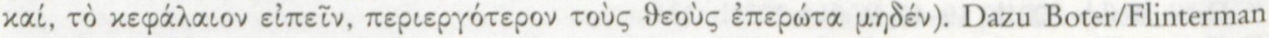
2007 , bes. $597 \mathrm{f}$.

${ }^{24}$ Graf 1999, 884. 
ser Nero angeblich viele Jahre lang nicht träumte. Träume waren an keinen Ort gebunden, sondern wurden dem Träumenden einfach zuteil. Dennoch gab es Möglichkeiten, Träume künstlich zu induzieren, etwa mit magischen Praktiken oder im Kontext der Inkubation, also des Schlafes im Heiligtum einer Gottheit. ${ }^{25}$

Wiewohl Träume als Alltagserfahrungen präsent gewesen sein dürften, bestand eine grundsätzliche Unsicherheit, nämlich ob der Traum signifikant war und für die Zukunft eine Relevanz besaß - oder eben nicht, denn antike Zeitgenossen machten immer wieder die Erfahrung, dass vor der Traumerfüllung nicht eindeutig darüber zu befinden war, welche Traumart vorlag, und dass sich die Deutung eines Traums dann noch nicht bewahrheitete. ${ }^{26}$ Um diese Unsicherheit zu minimieren, wurden Träume und verwandte Phänomene (z. B. Wachvisionen und Trugbilder) kategorisiert und auch Regelwerke für die Traumdeutung erarbeitet, die mit Interpretationen einzelner Traumsymbole und -szenerien aufwarteten. Professionelle Traumdeuter traten bei Festanlässen auf und es etablierte sich ein ,wissenschaftliches' Schrifttum, von dem sich nur die fünfbändigen Oneirokritika des Artemidor von Daldis aus der zweiten Hälfte des 2. Jh. n. Chr. erhalten haben. ${ }^{27}$ Allerdings: Mit der Deutung war die Unsicherheit nicht unbedingt behoben, denn die Interpretation desselben Traums durch verschiedene Traumdeuter musste kein identisches Ergebnis erbringen. ${ }^{28}$ Es gab nämlich keine übergeordnete, verbindliche Deutungsinstanz, sondern unterschiedliche Auslegungen desselben Symbols standen konkurrierend nebeneinander.

Die Träume einer Personengruppe stießen bei den antiken Zeitgenossen auf besonderes Interesse, nämlich von Königen und Kaisern. Diese Sichtweise ist schon in der Ilias beim genannten Traum des Agamemnon zu finden, auf den der echte Nestor antwortet: „Hätte irgendein anderer der Achaier diesen Traum

${ }^{25}$ Zur Inkubation bereitet Gil Renberg eine Monographie für den Druck vor; vgl. bereits Renberg 2006 und 2010. Zu den Zauberpapyri: Weber 2005-2006, 100-113.

${ }^{26}$ Vgl. dazu den Brief des Apollonios an Ptolemaios (Urkunden der Ptolemäerzeit I 70,6-13.28-30, zu datieren um 156 v. Chr.) aus dem Umfeld eines, Traumtagebuchs' in griechischer und demotischer Sprache: „Denn du lügst alles [zusammen] und die Götter bei Dir gleichfalls, denn sie haben uns in einen großen Schlamm geworfen und worin wir sterben können, und wenn du [sc. im Traum] gesehen hast, dass wir gerettet werden sollen, dann werden wir untergetaucht. ... in die Irre geführt von den Göttern im Glauben an die Träume." Auf der Vorderseite des Papyrus ist neben dem Adressaten

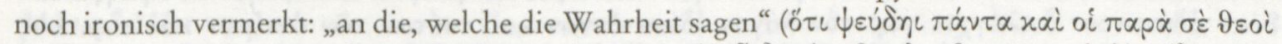

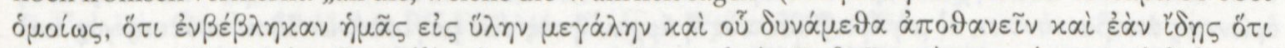

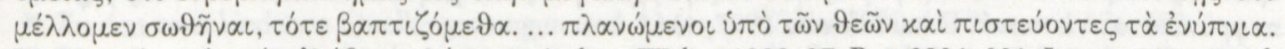

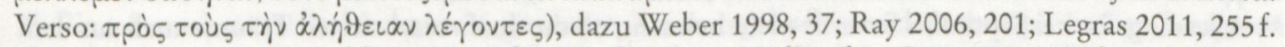
${ }^{27}$ Dazu Weber 1999a; Walde 2001a; außerdem die ersten Studien der ,Groupe Artémidore` in Montpellier: du Bouchet/Chandezon 2012. Artemidor ist zweifellos einzuordnen in die Gruppe der erst jüngst wieder stärker in das Blickfeld der Forschung gerückten Fach- bzw. Wissen(schafts)stexte (zu ihnen: Asper 2007 und Fögen 2009). Hinsichtlich seiner Präsentationsformen und -techniken besteht für Artemidor noch erheblicher Klärungsbedarf; methodisch weiterführend können hier die Ausführungen von Asper 2007 sein, der bei seiner Textauswahl Artemidor nicht berücksichtigt hat, dessen begriffliches Instrumentarium sich aber ohne weiteres auf die Oneirokritika übertragen lässt.

${ }^{28}$ Cicero, de divinatione 2,144-146. 
berichtet, Trug würden wir ihn nennen und uns lieber davon abwenden. Jetzt aber sah ihn der Mann, der sich weit der Beste rühmt unter den Achaiern. " 29 Artemidor geht in der methodischen Grundlegung seines Traumdeutungsschlüssels auf diesen Kontext ein: „Es ist nun vollends unmöglich, dass sich bei einem unbedeutenden Menschen entgegen seinen Fähigkeiten ein Traum über große Staatsangelegenheiten einstellt. ... Denn diese (sc. ein König oder hoher Beamter) haben die Aufgabe, für das Gemeinwesen Sorge zu tragen. "30 Damit war die Vorstellung verbunden, dass Könige und Kaiser, gesellschaftlich herausragende Personen überhaupt, zu den Göttern in einem besonderen Verhältnis standen, von ihnen eingesetzt oder begünstigt wurden. Der Zusammenhang zwischen den Träumen der Kaiser, deren Deutung und ihrer Erfüllung erfuhr deshalb besondere Beachtung, weil in ihrem Falle eine potentielle Relevanz für das Imperium bestand.

\section{Kaiser, Historiker und Biographen}

Cassius Dios kleine Schrift stellt vermutlich einen Sonderfall dar - zumindest hat sich kein vergleichbares Werk aus der Antike überliefert. Hingegen liegen Belege dafür vor, dass Principes wie Septimius Severus eigene Träume und solche, die sie betrafen, selbst aufgeschrieben bzw. gesammelt haben. ${ }^{31}$ Ein Großteil des überlieferten Traummaterials für Octavian/Augustus dürfte auf die Autobiographie zurückgehen, die der erste Princeps im Jahre 23 v. Chr. verfasste. ${ }^{32}$ Sueton weiß von einem ausgeprägten Interesse des Augustus:

Träumen maß er eine große Bedeutung bei, und zwar sowohl seinen eigenen als auch denen anderer, wenn er in ihnen vorkam. ... Während des ganzen Frühjahrs hatte er sehr viele überaus angsteinflößende, aber inhalts- und folgenlose Träume, in den übrigen Jahreszeiten träumte er seltener und weniger Unsinniges. ${ }^{33}$

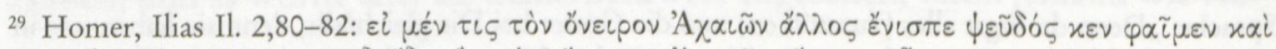

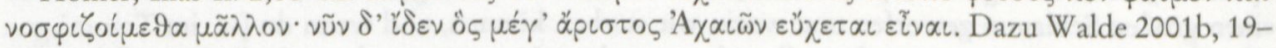
42.

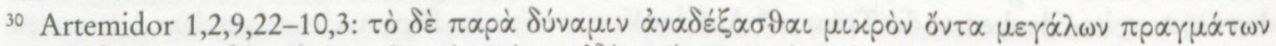

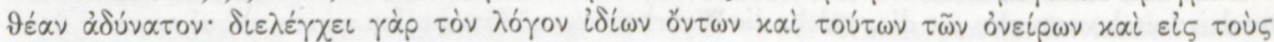

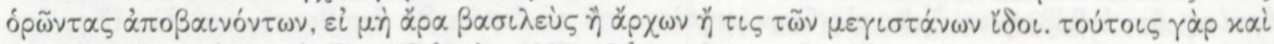

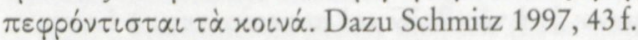

31 Allerdings ist bei Septimius Severus nicht bekannt, ob die dort verarbeiteten Träume auch aus dem Büchlein von Cassius Dio stammten und wie sehr Dio und Herodian dann wiederum dieses kaiserliche Material in ihre Geschichtswerke einbezogen haben.

${ }^{32}$ Bringmann/Wiegandt 2008, 191-215, und Smith 2009 mit den entsprechenden Textpassagen; umfassend dazu Weber 2003a und Wiseman 2009, außerdem - mit einer weiterführenden gattungsgeschichtlichen Einordnung - Pausch 2004, 311-314.

${ }_{33}$ Sueton, Augustus 91,1f.: somnia neque sua neque aliena de se neglegebat. ... ipse per omne ver plurima et formidulosissima et vana et irrita videbat, reliquo tempore rariora et minus vana. Dazu Weber 2000, 202 f.; Weber 2003a, 297 f. 
Sollte sich der letzte Teil der Bemerkung einem authentischen Diktum des Princeps verdanken, spricht dies für eine längerfristige Beobachtung der eigenen Traumaktivität und für eine Urteilsfähigkeit über die Signifikanz der Träume auf Seiten des Augustus. Geht man zeitlich weiter zurück, finden sich bei Mithridates VI., ca. 120-63 v. Chr. König von Pontos, und bei Sulla (138-78 v. Chr.) bemerkenswerte Details, und zwar zu zwei diametral verschiedenen Umgangsweisen mit Träumen: Mithridates bewahrte Träume von sich und seinen Frauen sowie Deutungen in einem Geheimarchiv auf, das dann Pompeius in die Hände fiel - offenkundig sollte das Material nicht an die Öffentlichkeit und damit in Umlauf gelangen, weil es als potentiell gefährlich angesehen wurde. Sulla schrieb in seinen im Alter verfassten commentarii über eigene Träume sowie deren Bedeutung für sein Leben und hat zuvor wohl auch mündlich darüber berichtet. ${ }^{34}$ Für andere Principes, etwa Hadrian, lassen sich derartige Zusammenstellungen nur vermuten. ${ }^{35}$ Wir haben freilich keine der genannten Schriften separat vorliegen, sondern nur Fragmente bei Historikern und Biographen, was zu einem weiteren Punkt führt.

Denn dem Interesse der Principes an den Träumen entsprach die große Aufmerksamkeit bei antiken Autoren: Für die frühe und hohe Kaiserzeit sind die Biographien von Plutarch und Sueton mit reichhaltigem Material, außerdem historische Werke, etwa von Flavius Josephus, Cassius Dio, Herodian und die Historia Augusta zu nennen. Selbst ein Rationalist wie Tacitus verschloss sich dem Genre nicht, und Valerius Maximus mit seiner exempla-Sammlung zur römischen Geschichte hielt ebenso Traummaterial bereit wie Mirabiliensammlungen, die sich im Umlauf befanden. ${ }^{36}$

Hier wird ein gravierendes Problem virulent: Nur selten lässt sich sicher sagen, ob der Bericht eines Traumes auf authentisches Traumerleben eines Kaisers zurückging oder ob der Kaiser selbst, eine Person aus seinem Umfeld oder der antike Autor ihn erfunden hat. ${ }^{37}$ Denn Träume waren in Gestalt und Motivik so vielfältig, dass sich keine stichhaltigen Kriterien zur Entscheidung in der genann-

\footnotetext{
${ }^{34}$ Plutarch, Pompeius 37,3 \& Sulla 37,1, dazu Weber 1999b, 21-24; 2000, 122-124; Wiseman 2009, 111 und 118.

35 Vgl. Dio Cassius 69,11,3 \& PG IV 2446-49, dazu Weber 2000, $106 \mathrm{f}$.

${ }^{36} \mathrm{Zu}$ den verschiedenen Quellengattungen und Autoren: Weber 2000, 65-91 sowie 576-585.

${ }^{37}$ Eine Ausnahme stellt das Anm. 26 genannte ,Traumtagebuch' aus dem memphitischen Serapeion dar, das aus einem Papyruskonvolut - die Texte gehören in die Jahre 168-152 v. Chr. - stammt. Es war nicht zur Publikation bestimmt; die Träume weisen keine Anzeichen einer literarischen Überformung auf, sondern wurden offenkundig direkt aufgezeichnet. Die Auswertung dieser Träume steht deswegen vor größeren Schwierigkeiten, weil die Träumenden teils dem griechischen, teils dem ägyptischen Milieu entstammten und bei vermuteten Tagesresten nicht die Möglichkeit besteht, die Informationen mit verlässlichen biographischen Details zu korrelieren; die moderne Forschung hat diese aufschlussreichen Texte erst in jüngster Zeit wieder ,entdeckt', dazu Weber 1998, $29 \mathrm{ff}$.; Legras 2005; Bresciani 2005, 125-139; Ray 2006.
} 
ten Frage aufstellen lassen ${ }^{38}$ - etwa eine besondere Logik oder besondere Wirrnis, wiewohl man hier mit Plausibilitäten arbeiten muss.

Warum aber sollten Träume erfunden werden? Sie stellten Möglichkeiten dar, mit Blick auf den Traumausgang eine Öffentlichkeit oder Teile derselben in eine bestimmte Richtung zu beeinflussen, was unter anderem ein byzantinischer Beleg explizit macht. ${ }^{39}$ Sie dienten aber auch als gestalterische Mittel für die Autoren, ${ }^{40}$ um Spannung zu erzeugen, um Ereignisse zu erklären, um Herrscher zu charakterisieren. ${ }^{41}$ Dennoch ist davon auszugehen, dass die Autoren in erheblichem Maße entsprechende Informationen in der Überlieferung oder bei befragten Zeitgenossen vorfanden. Dabei wurden offenkundig Träume einem Selektionsprinzip unterworfen: Aufgenommen in die biographischen und historiographischen Werke wurde nur, was im Hinblick auf einen Staat oder den Kaiser relevant erschien. Träume anderer Personen mit niedrigerem Sozialstatus fanden hingegen nur dann Berücksichtigung, wenn sie direkt oder indirekt auf die genannten Themen bezogen waren. Dies führt zu der Frage, in welchen politischen Kontexten Träume überhaupt zu finden waren.

\section{Kontexte, Publikation und Unterdrückung}

Das Material an Träumen von und für römische Kaiser lässt sich in sechs Rubriken untergliedern: die Geburt und Kindheit, die Verheißung der Herrschaft, die Erringung eines Sieges und das göttliche Eingreifen in der Schlacht, die Ausübung der Herrschaft, die besondere Befähigung und göttliche Begünstigung sowie das nahende Ende. Aus den zahlreichen Beispielen seien drei herausgegriffen, bei denen die politische Dimension in besonderem Maße zutage tritt.

a. Sueton und Cassius Dio berichten mit großer Übereinstimmung einen Traum, der Vespasian zugeschrieben wurde:

\footnotetext{
38 Anders Harris 2009.

${ }^{39}$ Hier ist ein allerdings später Beleg, aus den Taktika (20,170 [PG 107,1061] des byzantinischen Kaisers Leon VI. (886-912) wichtig: „[Träume] zu erfinden und die Soldaten zu überreden, damit sie deinen Träumen, die den Sieg ankündigen, glauben, ist besonders zum Zeitpunkt des Krieges

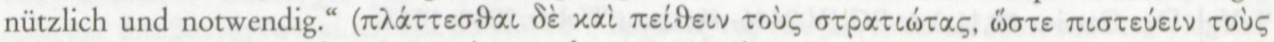
бoù

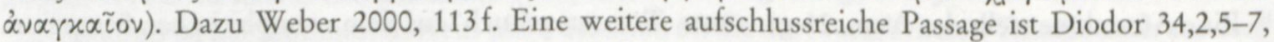
dazu Weber 2000, $179 \mathrm{f}$.

${ }^{40}$ Dies gilt auch für andere literarische Gattungen, dazu Walde 2001b, passim.

${ }^{41}$ Es ist auch evident, dass man sich mit der Berufung auf Träume keineswegs der Lächerlichkeit preisgab, sondern sich gerade die Elite, hier die Bevölkerung in der römischen Kaiserzeit, intensiv mit ihnen beschäftigte. Plinius der Jüngere diskutierte etwa in einem Brief einen Traum des Biographen Sueton und stellte Überlegungen darüber an, inwieweit man sich davon beeindrucken lassen sollte (dazu Weber 2000, 68-71). Es war also kaum verwunderlich, wenn durch Träume in das individuelle Leben eingegriffen wurde.
} 
Ein Traum aber offenbarte ihm, wenn Caesar Nero einen Zahn verliere, werde er selber Kaiser sein. Diese Prophezeiung mit dem Zahn wurde schon am nächsten Tag Wirklichkeit. ... Dieses Vorzeichen verlangte eine Deutung. ${ }^{42}$

Es handelt sich, zumal kein Gott agiert, um ein eher unspektakuläres Traumbild. Allerdings ist das Motiv des Zahnverlustes ebenso in früheren Herrscherträumen signifikant, und auch Artemidor widmete den Zähnen ein ganzes Kapitel. ${ }^{43}$ Hier aber besteht eine klare Diskrepanz zwischen der Erfüllung des Traumes und der tatsächlichen Chronologie des Herrschaftsübergangs: Der Traum zu Neros Lebzeiten überbrückte die Wirren des Vierkaiserjahres und ließ den Sieg Vespasians als vorherbestimmt erscheinen. Für eine tatsächliche Verbreitung dieses Traumes durch Vespasian haben wir weder positive noch negative Evidenz, sondern es handelt sich am ehesten um eine Erfindung nach Neros Tod bzw. während der Aufbauphase von Vespasians Herrschaft.

b. Tacitus überliefert in seinen Annales einen Traum im Kontext eines Prozesses unter Kaiser Claudius; er sei stellvertretend für etliche Fälle angeführt, in denen Träume und ihre Deutung zum Tod der Beschuldigten führten:

Darauf beruft man den Senat, und Suillius fordert weiter zwei erlauchte Ritter namens Petra vor Gericht. Der Grund für ihre Beseitigung lag darin, dass sie ihr Haus für die Zusammenkünfte Mnesters mit Poppaea zur Verfügung gestellt haben sollten. Freilich wurde dem einen auch ein nächtlicher Traum vorgeworfen: Er habe Claudius mit einem Ährenkranz geschmückt gesehen, doch mit rückwärts gerichteten Ähren, und aus dieser Erscheinung eine drückende Teuerung vorhergesagt. Manche berichteten, einen Kranz aus Weinlaub mit vergilbten Blättern habe er gesehen und so gedeutet, dass für das Ende des Herbstes der Tod des Princeps angekündigt werde. So viel steht außer jedem Zweifel, dass irgendein Traum ihm selbst und seinem Bruder Verderben gebracht hat. ${ }^{44}$

Es handelt sich wohl um zwei Varianten desselben Traums, die Tacitus vermutlich in verschiedenen Quellen vorgefunden hatte und mit denen er sich als „gewissenhafter und vertrauenswürdiger Berichterstatter erweisen " kann, ${ }^{45}$ ohne

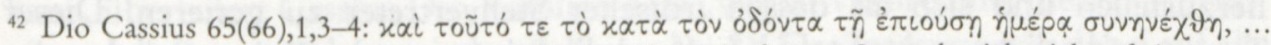

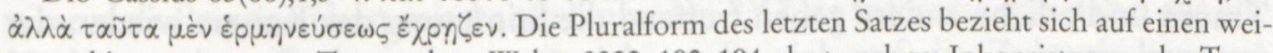
teren, hier ausgesparten Traum, dazu Weber 2000, 190-194, dort auch zu Inkonsistenzen des Traumes, außerdem Vigourt 2001, 358.

${ }^{43}$ Herodot 6,107,1-108,1 (Hippias), dazu Weber 2000, $181 \mathrm{f}$; Dionysios Halikarnassos 20,12 (Pyrrhos), dazu Weber 1999b, 15, Anm. 48; Artem. 1,31 und 2,67.

${ }^{44}$ Tacitus, Annales 11,4,1-2: vocantur post haec patres, pergitque Suillius addere reos equites Romanos inlustres, quibus Petra cognomentum. at causa necis ex eo quod domum suam Mnesteris et Poppaeae congressibus praebuisset. verum nocturnae quietis species alteri obiecta, tamquam vidisset Claudium spicea corona evinctum spicis retro conversis, eaque imagine gravitatem annonae dixisset. quidam pampineam coronam albentibus foliis visam atque ita interpretatum tradidere, vergente autumno mortem principis ostendi. illud haud ambigitur, qualicumque insomnio ipsi fratrique perniciem allatam. Dazu Weber 2000, 333-335; Vigourt 2001, 283-285; bes. Hausmann 2009, 168-174, mit weiter führenden Beobachtungen zur sprachlichen Gestaltung und zum Kontext des Prozesses. Zum weiteren historischen Hintergrund: Osgood 2011, 147-151.
}

${ }^{45}$ Hausmann 2009, 171. 
den genauen Details zu große Bedeutung zuzumessen. Die Ähren wurden von den Zeitgenossen des Claudius, auf dessen Kopf sich das Symbol befand, mit der Aufgabe des princeps zusammengebracht, für die Getreideversorgung Roms zu sorgen. Aus den rückwärts gerichteten Ähren ließ sich glaubhaft machen, dass eine Getreideknappheit, Hungersnot und Teuerung bevorstanden. Das Problem für die Brüder bestand nicht in den Träumen, sondern in der Deutung, die wohl gerüchteweise öffentlich gemacht wurde; zieht man den weiteren Kontext der Episode heran, wird deutlich, dass es in Wirklichkeit „um etwas ganz Anderes ging als um die hier vor dem Senat verhandelte Anschuldigung “. ${ }^{46}$ Dennoch: Politisch brisant werden konnte schlicht alles, was mit dem Kaiser in Verbindung stand, aber dessen Kontrolle entzogen war. Eine maiestas-Klage ließ sich immer aktivieren, insbesondere bei den Themen, Getreideversorgung' und ,Voraussage des Todes eines Kaisers', ${ }^{47}$ zumal Gerüchte angesichts der gesamten Kommunikationssituation dazu prädestiniert waren, Unruhen und Aufstände zu provozieren. ${ }^{48}$ Wahrscheinlich wurden die Brüder Petra als Urheber der Verbreitung verantwortlich gemacht und es war ihnen nicht möglich, sich von dem Verdacht zu befreien. Die Träume und die Deutungen ließen sich als reale Agitation gegen den Princeps verstehen.

c. In seiner Domitian-Vita berichtet Sueton einen Traum, der auf das nahende Ende des Kaisers verwies und den auch Cassius Dio in modifizierter Form überliefert: „Auch träumte ihm, Minerva, die er abergläubisch verehrte, verlasse ihr Heiligtum und erkläre, sie könne ihn nicht mehr schützen, da sie von Iuppiter entwaffnet worden sei." 49

Angespielt wird auf Minervas Funktion als Schutzgöttin Domitians, ein Traummotiv, das auch für Galba und Fortuna bekannt ist. Minerva steht in Abhängigkeit von Iuppiter und muss der höheren Macht gehorchen, auf deren Weisung die Beendigung des Schutzverhältnisses erfolgt ist, da es um das Wohl des ganzen Staates geht. Dies ist umso bezeichnender, als sich Domitian bemüht hat, auf Münzen und in weiteren Inszenierungen eine Verbindung zu Iuppiter herzustellen und sich als dessen irdischer Stellvertreter zu gerieren. Dieser Traum und etliche andere geben treffend die Stimmung des Kaisers wieder, der

46 Hausmann 2009, 172.

47 Vgl. Gizewski 1999, 711 f.; Graf 1999, 886; exemplarisch für die Grundprinzipien solcher Klagen und den sozialen Kontext: Flaig 2003. Hausmann 2009, 171 f., Anm. 472, macht freilich zu Recht deutlich, dass Versuchen, hier konkrete historische Begebenheiten finden zu wollen, auf die die Träume vorausdeuten sollten, kein Erfolg beschieden ist.

${ }^{48}$ Zur großen Bedeutung von Gerüchten, auch für die (stadt-)römische Gesellschaft der Kaiserzeit: Kuhn 2012, 18-20. Bisher einschlägig ist Ries 1969, allerdings auf Tacitus begrenzt. Für die griechische Literatur von Homer bis Polybios jetzt Larran 2009.

49 Sueton, Domitian 15,3: Minervam, quam superstitiose colebat, somniavit excedere sacrario negantemque ultra se tueri eum posse, quod exarmata esset a Iove. Dazu Weber 2000, 456-458, dort auch zur ausführlicheren Version bei Cassius Dio; Vigourt 2001, 329 und passim. 
angsterfüllt den Tod erwartet - zumindest hat man sich dies im Nachhinein so vorgestellt. ${ }^{50}$

Die Beispiele rühren an die grundsätzliche Frage nach den Umständen der Publikation bzw. deren Unterdrückung. ${ }^{51}$ Sieht man von den zuvor genannten kaiserlichen Schriften, in denen Träume enthalten waren, und von der für Septimius Severus bezeugten bildlichen Umsetzung seiner Träume einmal ab, gab es sicherlich nur in Ausnahmefällen schriftliche Unterlagen darüber, etwa in den acta diurna. Man hat vor allem mit einer mündlichen Verbreitung zu rechnen. In bestimmten Situationen dürften die Kaiser selbst ein Interesse an der Verbreitung entsprechender Traumdetails gehabt haben, wie es für das Ausstreuen von Gerüchten unter den Soldaten während des Konflikts von Octavian und Marcus Antonius bekannt ist: ${ }^{52}$ Träume mit einer günstigen Deutung bzw. Erfüllung wie Vespasians Traum konnten positiv auf die nähere und weitere Umgebung einwirken, die Stellung des Princeps festigen und Handlungen plausibel machen auch erst im Nachhinein. Allerdings dürfte es schwierig gewesen sein, die Verbreitung negativ konnotierter Träume zu verhindern, wenn ein Kaiser sie überhaupt anderen kommunizierte, worauf noch einzugehen ist. Obwohl etliche Passagen suggerieren, dass aus dem kaiserlichen Umfeld nur wenige Informationen nach außen gelangten, die dazu noch schwer nachprüfbar waren, hatten doch viele Personen in unterschiedlichen Kontexten mit der Zentrale zu tun, und es gab auch einzelne, die ein großes Wissen über den Kaiser besaßen. Verschiedene antike Autoren, die dieses Thema besonders interessierte, suggerieren implizit oder explizit einen steten Fluss von angeblichen Fakten, Gerüchten und Halbwahrheiten vom Kaiserhof nach außen. ${ }^{53}$

Ein Geheimarchiv wie das von Mithridates, mit dem vermutlich die Verbreitung ungünstiger oder strittiger Deutungen verhindert werden sollte, war für Rom kaum denkbar. Eine der wenigen Passagen überhaupt, aus der man eine Verbreitung ungünstiger Trauminhalte - hier die Alpträume Caracallas -, ausgehend vom höfischen Ambiente, noch zu Lebzeiten des Princeps schließen kann, überliefert Cassius Dio, nämlich dass „wegen Verbreitung dieser Geheimnisse viele Personen hart misshandelt wurden. “54 Das Interesse war dabei auf alle Vorkommnisse, Ereignisse usw. gerichtet, die sich auf die Zukunft bezogen

${ }^{50}$ Eine treffende Analyse der biographischen Forschung zu Domitian findet sich bei Schnurbusch 2011, 286-289 und 292-294.

${ }^{51}$ Für das Folgende: Weber 2000, 120-133.

52 Eich 2000, 273-275 mit Belegen.

53 Dazu Weber 2000, 129-123, mit Belegen und weiterer Literatur; zu den Informationen, die Cassius Dio zur Verfügung standen: Moscovich 2004, $358 \mathrm{ff}$. Als konkrete Orte der Verbreitung könnten Prozessionen, das Forum, Familien- und Klientelbeziehungen, kaiserliche Reisen und sicherlich auch Briefe gedient haben. Hausmann 2009, 108-111 und passim, kann an verschiedenen Passagen aufzeigen, wie zentral „die Wiedergabe von Gerüchten ... für die Kunst der Leserlenkung ist.“

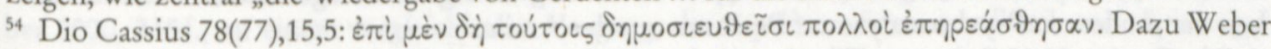
2000, 463-465; Weber 2011, $122 \mathrm{f}$. 
haben - sei es zur Legitimierung von Handlungen und Ansprüchen, sei es zur Diskreditierung derselben und zweifellos auch zur Beeinflussung zukünftigen Geschehens: Dieses Interesse, verbunden mit der besonderen Stellung der jeweiligen Person, stellt die entscheidende Voraussetzung dafür dar, dass Zeichen, Gerüchten etc. so viel Glauben entgegengebracht wurde..$^{5}$ Allerdings dürfte die Publikation vor der Erfüllung einer Traumdeutung eher die Ausnahme gewesen sein. Danach konnte die Begebenheit in die biographische und historiographische Literatur Eingang finden, durchaus in konkurrierenden Versionen.

\section{Deuter, Deutung und Wissen}

Es war bereits davon die Rede, dass einige Träume, die verschlüsselten, der Deutung bedurften, andere eben nicht. Eine für unser Thema zentrale Frage ist deshalb, wer die Träume der Kaiser deutete und auf welcher Wissensbasis dies geschah. ${ }^{56}$ Sulla, Augustus und Septimius Severus sammelten offenkundig ihre Träume und dürften eine gewisse Deutungskompetenz entwickelt haben. ${ }^{57}$ Worauf sie basierte und wie sie angeleitet war, ist nicht überliefert, aber es existierten zweifellos schriftliche Traumdeutungsschlüssel und Auseinandersetzungen mit dem Phänomen einschließlich etlicher Beispiele. ${ }^{58}$ Marc Aurel formulierte in seiner Autobiographie, ihm seien „durch Träume Hilfsmittel zuteil geworden, unter anderem gegen Blutspucken und Schwindelanfälle“, und war sowieso der Überzeugung, dass die Götter den Menschen Träume als Hilfe sandten. ${ }^{59}$ Außerdem waren manche Principes, etwa Tiberius, ausgewiesene Experten für andere Formen der Divination. ${ }^{60}$

Für die Principes sind etliche Namen von Wahrsagern, Sehern, Astrologen und Magiern bekannt, die konsultiert wurden; es muss aber auch Traumdeuter am Kaiserhof gegeben haben. Freilich bleibt diese Gruppe anonym. So wurde etwa Caesar von coiectores zu größeren Hoffnungen ermutigt, aber wir kennen

\footnotetext{
${ }_{55}$ Zur Vorherbestimmtheit der kaiserlichen Herrschaft im Kontext astrologischer Praktiken: Le Bouffle 1999.

${ }^{56}$ Für das Folgende: Weber 2000, 99-120.

57 Jenseits der drei genannten Principes liegt kein entsprechendes Material vor, wobei man davon ausgehen muss, dass der Bildungsstand mancher Soldatenkaiser sowieso zu wünschen übrig ließ, auch wenn „das Herrscherhaus weiterhin für Bildungsdemonstrationen empfänglich war", so Pietzner 2008, 890, außerdem 873-875. Zum Bildungsstand von Septimius Severus: Spielvogel 2006, 19-23 und $50-52$.

58 Dazu bes. Vinagre Lobo 2011, 95-137.

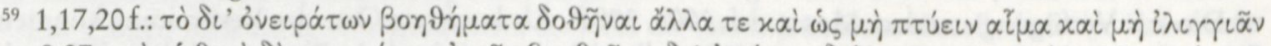

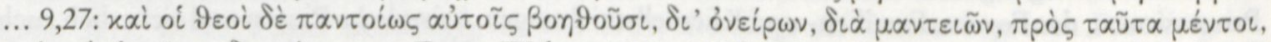

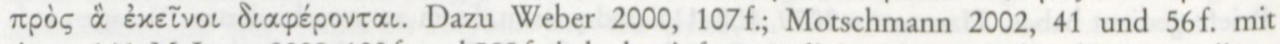
Anm. 146; McLynn 2009, 103 f. und 589f., jedoch mit fragwürdigen Aussagen über die Anwendbarkeit der modernen Psychoanalyse auf Personen aus der Antike.
}

${ }^{60}$ Dio Cassius 57,15,7, Sueton, Tiberius 14, dazu Weber 2000, $103 \mathrm{f}$. 
weder deren Namen und Anzahl noch ihre Aufenthaltsdauer bei Caesar. ${ }^{61} \mathrm{Ge}-$ spräche mit Traumdeutern sind sonst nur für spätantike Kaiser bekannt, weshalb die Quellen den Eindruck vermitteln, dass ein solches Vorgehen eher die Ausnahme gewesen war. Auch haben wir nur aus der Spätantike Belege dafür, dass ein Kaiser eigene Träume mit seiner engeren Umgebung, mit Familie und Freunden, besprach. ${ }^{62}$ Etliche dieser Träume geben sich als direkte Ankündigungen, die die Kaiser stets befolgten, aber es ist nicht auszuschließen, dass auch darüber ein Deuteprozess in Gang gesetzt wurde.

Gemeinsames Deuten und Diskutieren der Träume war den antiken Autoren zufolge nicht die Regel - wohl einfach deswegen, weil ein großes Risiko bestand. Der Kaiser hatte sich stets zu fragen, ob er den Deutern trauen konnte bzw. diese dem Kaiser ihre eigentliche, möglicherweise ungünstige Interpretation überhaupt übermittelten, zumal wenn derselbe Traum auch anders gedeutet werden konnte. Die Deuter zogen sich einem Zeugnis des spätantiken Historikers Ammian zufolge bestenfalls den Unwillen des Kaisers zu: Kaiser „Iulian musste immerhin befürchten, dass man sich Antworten ausdachte, die seinen eigenen Wünschen entgegenkamen, und war deswegen verärgert. "63 Auch ein kaiserlicher Prokurator, der Fulvius Plautianus, dem Prätorianerpräfekten und Vater von Caracallas Frau Publia Fulvia Plautilla, einen Traum auf die Übernahme der Herrschaft hin gedeutet haben soll, geriet in eine prekäre Lage. ${ }^{64}$ Nahm ein Princeps seine Träume und deren Botschaft ernst, war er gut beraten, wenn er sich in seiner Kommunikation mit dem Göttlichen nicht von anderen abhängig machte. Denn sonst gesellte sich zur vorhandenen Unsicherheit die Angst vor einer Verselbständigung des Geschehens und vor negativen Folgen aus der Deutung hinzu. Andererseits standen bei einem Versagen der Deutung mit den Deutern ,Sündenböcke‘ bereit, die jeden Prestigeverlust vom Kaiser abwenden konnten.

Welche Träume ein Princeps überhaupt mitteilte, wird er von seiner Einschätzung der Deutung und auch von der politischen und privaten Gesamtsituation abhängig gemacht haben. Da Träume vielfach mit Krisen- und Entscheidungssituationen verbunden waren und somit auch für die Angst stehen, der ein Kaiser ausgesetzt war, lässt sich kaum davon ausgehen, dass nur diejenigen Träume nach außen drangen, deren Deutung für den Kaiser günstig erschienen: Gerade

${ }^{61}$ Dazu Weber 2000, 115-120.

${ }^{62} \mathrm{Zu}$ den Beraterkreisen um den Princeps generell: Weber 2000, 127-129; für die Severerzeit: Laeben-Rosén 2005, 136-170.

${ }^{63}$ Ammianus Marcellinus 22,1,2: cumque ille timeret ne cupiditati suae congruentia fingerentur, atque ideo maestus, dazu Weber 2000, 119, Anm. 142, mit weiteren Hinweisen.

${ }_{64}$ Zu Plautianus, Aelius Koiranos und Dio Cassius 77(76),5,4: Freyburger-Galland 1999, 540 f.; Weber 2000, $345 \mathrm{f}$; zu einem vergleichbaren Vorgang, der Septimius Severus selbst betraf: Spielvogel 2006, $54 \mathrm{f}$. 
in solchen Situationen war der Gesprächsbedarf am größten, doch wird dies durch den Quellenbefund nicht bestätigt.

\section{Zusammenfassung: Träume und Wissen über die Zukunft}

Was meint nun im Kontext dieser politisch relevanten Träume Wissen über die Zukunft? Zunächst handelt es sich bei den Träumen um potentielles Vorauswissen über den Ausgang einer Situation, gekoppelt mit einer möglichen Handlungsanweisung, auch zur Vermeidung bestimmter Ereignisse. Wichtig ist die Unterscheidung zwischen direkten Traumanweisungen und verschlüsselten Botschaften, die der Deutung bedurften: Erstere kamen direkt von den Göttern, während für die Deutung die Wahl des richtigen Sozialpartners ein Problem darstellen konnte. Denn die ,richtige' Deutung setzte ein erhebliches Wissen sowohl über die Signifikanz einzelner Symbole und der Deuteregeln als auch praktische Erfahrung voraus; allerdings waren konkurrierende Deutungen desselben Traumes möglich, da viele Symbole kulturell polyvalent kodiert waren. Für das Agieren eines Kaisers im politischen Raum erwies sich das Wissen des Deuters als prekär, weil der Princeps sich in die Abhängigkeit eines Deuters begab und Gefahr lief, dass sein Traum verbreitet und eben anders gedeutet wurde. Diese Problemlage erschien umso virulenter, als Träume meist mit Entscheidungssituationen verbunden waren: Hier wird deutlich, dass Traumwissen, so fragwürdig es gewesen sein mag, Macht bedeutete. Denn die Unsicherheit, die der Träumende mit der Deutung zu reduzieren suchte, ließ sich potentiell auch gegen ihn wenden. Hierin dürfte der Grund dafür liegen, dass wenig von Deutern kaiserlicher Träume bekannt ist und vielmehr einige Principes selbst die Deutung vornahm was nicht verhinderte, dass unautorisiertes Material durchaus in Umlauf kam. Träume anderer, die den Kaiser zum Inhalt hatten, konnten für den Träumenden und für den in diesem Punkt ohnmächtigen Kaiser strukturell gefährlich werden, denn Träume entzogen sich letztlich der Kontrolle, auch der gesetzlich verordneten.

Allerdings - und dies ist nachdrücklich zu unterstreichen: In etlichen Fällen legt sich der Eindruck nahe, dass Angst- und Alpträume der postumen Abrechnung dienten, es wurden aber auch andere Träume eingeführt, somit also erfunden - von wem auch immer. Damit handelt es sich nicht um ein Wissen über die Zukunft in der konkreten historischen Situation, sondern um eine Rückprojektion ex eventu. Ein solches Vorgehen war freilich nur möglich, weil Träume als Träger zukünftigen Wissens angesehen wurden. 


\section{Bibliographie}

Asper, Markus: Griechische Wissenschaftstexte. Formen, Funktionen, Differenzierungsgeschichten, Stuttgart 2007.

Birley, Anthony Richard: The African Emperor Septimius Severus, London 1988.

Boter, Gerard/Flinterman, Jaap-Jan: Are Petitionary Dreams Non-Predictive? Observations on Artemidorus' Oneirocritica 1.6 and 4.2, in: Mnemosyne 60, 2007, 589-607.

Bresciani, Edda: La porta dei sogni. Interpreti e sognatori nell'Egitto antico, Turin 2005.

Bringmann, Klaus/Wiegand, Dirk (Hg.): Augustus. Schriften, Reden und Aussprüche, Darmstadt 2008.

Brodersen, Kai (Hg.): Prognosis. Studien zur Funktion von Zukunftsvorhersagen in Literatur und Geschichte seit der Antike, Münster 2001.

Chausson, François: L'autobiographie de Septime Sévère, in: Revue des Études Latines 73, 1995, 183-198.

du Bouchet, Julien/Chandezon, Christophe (Hg.): Études sur Artémidore et l'interprétation des rêves, Nanterre 2012.

Eich, Armin: Politische Literatur in der römischen Gesellschaft. Studien zum Verhältnis von politischer und literarischer Öffentlichkeit in der späten Republik und frühen Kaiserzeit, Köln 2000.

Flaig, Egon: Können wir den Majestätsprozeß gegen C. Silius (24 n. Chr.) verstehen? oder Wir verstehen nur, was erklärt ist, in: Marlies Heinz/Manfred K. H. Eggert/Ulrich Veit (Hg.): Zwischen Erklären und Verstehen? Beiträge zu den erkenntnistheoretischen Grundlagen archäologischer Interpretation, Münster 2003, 23-52.

Fögen, Thorsten: Wissen, Kommunikation und Selbstdarstellung. Zur Struktur und Charakteristik römischer Fachtexte der frühen Kaiserzeit, München 2009.

Frede, Dorothea: Art. Schicksal, in: Der Neue Pauly 11, 2001, 156-158.

Freyburger-Galland, Marie-Laure: Les rêves chez Dion Cassius, in: Revue des Études Anciennes 101, 1999, 533-545.

Gil, Luis: La diagnosis onirica en Galeno, in: Cuadernos de filologia clásica. Estudios griegos e indo-europeos 14, 2004, 139-153.

Gizewski, Christian: Art. Maiestas, in: Der Neue Pauly 7, 1999, 710-712.

Graf, Fritz: Art. Divination/Mantik, in: Religion in Geschichte und Gegenwart ${ }^{4}$ 2, 1999, 883-886.

Handy, Markus: Die Severer und das Heer, Berlin 2009.

Harris, William V.: Dreams and Experience in Classical Antiquity, Cambridge, Mass./ London 2009.

Hausmann, Michael: Die Leserlenkung durch Tacitus in den Tiberius- und Claudiusbüchern der ,Annalen', Berlin 2009.

Holowchak, M. Andrew: Interpreting Dreams for Corrective Regimen. Diagnostic Dreams in Greco-Roman Medicine, in: Journal of the History of Medicine 56, 2001, 382-399.

Hose, Martin: Cassius Dio: A Senator and Historian in the Age of Anxiety, in: John Marincola (Hg.): A Companion to Greek and Roman Historiography 2, Oxford 2007, 461-467.

Kuhn, Christina: Politische Kommunikation und öffentliche Meinung in der antiken Welt. Einleitende Bemerkungen, in: dies. (Hg.): Politische Kommunikation und öffentliche Meinung in der antiken Welt, Stuttgart 2012, 11-30. 
Kuhn-Chen, Barbara: Geschichtskonzeptionen griechischer Historiker im 2. und 3. Jahrhundert n. Chr. Untersuchungen zu den Werken von Appian, Cassius Dio und Herodian, Frankfurt am Main 2002.

Kuhlmann, Peter A.: Die Macht des Numinosen in der Welt. Prodigien und Götterwille in Rom, in: Reinhard G. Kratz/Hermann Spieckermann (Hg.): Vorsehung, Schicksal und göttliche Macht. Antike Stimmen zu einem aktuellen Thema, Tübingen 2008, 171-192.

Larran, Francis: Le bruit qui vole. Histoire de la rumeur et de la renommée en Grèce ancienne, Toulouse 2011.

Laeben-Rosén, Viktoria: Age of Rust. Court and Power in the Severan Age (188-238 AD), Uppsala 2005.

Le Bœuffle, André: Le pouvoir et la ,rétro-prédestination“, ou l'art de la déformation ... astrologique, in: Élisabeth Smadja/Évelyne Geny (Hg.): Pouvoir, divination, prédestination dans le monde antique, Besançon 1999, 273-282.

Legras, Bernard: Droit et culture dans le Sarapieion de Memphis: Les rêves d'Apollonios fils de Glaukias, in: Robert W. Wallace/Michael Gagarin (Hg.): Symposion 2001. Vorträge zur griechischen und hellenistischen Rechtsgeschichte, Wien 2005, 223-236.

Legras, Bernard: Les reclus grecs du Sarapieion de Memphis. Une enquête sur l'hellénisme égyptien, Löwen 2011.

Lichtenberger, Achim: Severus Pius Augustus. Studien zur sakralen Repräsentation und Rezeption der Herrschaft des Septimius Severus und seiner Familie (193-211 n. Chr.), Leiden/Boston 2011.

Liatsi, Maria: Zur Funktion des Traumes in der antiken Medizin (Hippokrates, De victu IV), in: Jochen Althoff/Bernhard Herzhoff/Georg Wöhrle (Hg.): Antike Naturwissenschaft und ihre Rezeption 12, Trier 2002, 7-21.

McLynn, Frank: Marcus Aurelius. Warrior, Philosopher, Emperor, London 2009.

Moscovich, M. James: Cassius Dio's Palace Sources for the Reign of Septimius Severus, in: Historia 53, 2004, 356-368.

Motschmann, Cornelius: Die Religionspolitik Marc Aurels, Stuttgart 2002.

Osgood, Josiah: Claudius Caesar. Image and Power in the Early Roman Empire, Cambridge 2011.

Pausch, Dennis: Formen literarischer Selbstdarstellung in der Kaiserzeit. Die von römischen Herrschern verfaßten autobiographischen Schriften und ihr literarisches Umfeld, in: Rheinisches Museum für Philologie 147, 2004, 303-336.

Pietzner, Katrin: Bildung, in: Klaus-Peter Johne (Hg.): Die Zeit der Soldatenkaiser. Krise und Transformation des Römischen Reiches im 3. Jahrhundert n. Chr. (235-284) 2, Berlin 2008, 863-892.

Ray, John: The Dreams of the Twins in St Petersburg, in: Kasia Szpakowska (Hg.): Through a Glass Darkly. Magic, Dreams and Prophecy in Ancient Egypt, Swansea 2006, 189-203.

Renberg, Gil H.: Was Incubation Practiced in the Latin West?, in: Archiv für Religionsgeschichte 8, 2006, 105-147.

Renberg, Gil H.: Dream-Narratives and Unnarrated Dreams in Greek and Latin Dedicatory Inscriptions, in: Emma Scioli/Christine Walde (Hg.): Sub Imagine Somni: Nighttime Phenomena in Greco-Roman Culture, Pisa 2010, 33-61.

Ries, Wolfgang: Gerücht, Gerede, öffentliche Meinung. Interpretationen zur Psychologie und Darstellungskunst des Tacitus, Heidelberg 1969.

Schmidt, Manfred G.: Die ,zeitgeschichtlichen' Bücher im Werke des Cassius Dio - von Commodus zu Severus Alexander, in: ANRW II 34,3, 1997, 2591-2649. 
Schmitz, Thomas A.: Bildung und Macht. Zur sozialen und politischen Funktion der zweiten Sophistik in der griechischen Welt der Kaiserzeit, München 1997.

Schnurbusch, Dirk: Rationalität und Irrationalität. Die Flavier in der Sicht der biographischen Forschung, in: Aloys Winterling (Hg.): Zwischen Strukturgeschichte und Biographie. Probleme und Perspektiven einer neuen Römischen Kaisergeschichte 31 v. Chr.-192 n. Chr., München 2011, 295-316.

Sidebottom, Harry: Severan Historiography: Evidence, Patterns, and Arguments, in: Simon Swain/Stephen Harrison/Jas Elsner (Hg.): Severan Culture, Cambridge 2007, $52-82$.

Smith, Christopher: The Memoirs of Augustus: testimonia and Fragments, in: Christopher Smith/Anton Powell (Hg.), The Lost Memoirs of Augustus and the Development of Roman Autobiography, Swansea 2009, 1-13.

Sordi, Marta: Le date di composizione dell'opere di Dione Cassio, in: Mario Capasso/ Sergio Pernigotti (Hg.), Studium atque urbanitas. Miscellanea in onore di Sergio Daris, Galatina 2001, 391-395.

Spielvogel, Jörg: Septimius Severus, Darmstadt 2006.

Starr, Raymond J.: The Circulation of Literary Texts in the Roman World, in: Classical Quarterly 37, 1987, 213-223.

Vigourt, Annie: Les Présages Impériaux d'Auguste à Domitien, Paris 2001.

Vinagre Lobo, Miguel Ángel: Los libros griegos de interpretación de sueños, Saragossa 2011.

Walde, Christine: Antike Traumdeutung und moderne Traumforschung, Düsseldorf/ Zürich 2001a.

Walde, Christine: Die Traumdarstellungen in der griechisch-römischen Dichtung, München/Leipzig 2001b.

Weber, Gregor: Traum und Alltag in hellenistischer Zeit, in: Zeitschrift für Religions- und Geistesgeschichte 50/1, 1998, 22-39.

Weber, Gregor: Artemidor und sein ,Publikum', in: Gymnasium 106/3, 1999a, 209-229.

Weber, Gregor: Herrscher und Traum in hellenistischer Zeit, in: Archiv für Kulturgeschichte 81/1, 1999b, 1-33

Weber, Gregor: Kaiser, Träume und Visionen in Prinzipat und Spätantike, Stuttgart 2000.

Weber, Gregor: Die Träume des Augustus, in: Gregor Weber/Martin Zimmermann (Hg.): Propaganda - Selbstdarstellung - Repräsentation im römischen Kaiserreich des 1. Jhs. n. Chr. Stuttgart 2003a, 297-316.

Weber, Gregor: „Zweifach sind die Tore der wesenlosen Träume ...“. Traum und Traumdeutung in der Antike, in: Thomas Auchter/Michael Schlagheck (Hg.): Theologie und Psychologie im Dialog über den Traum, Paderborn 2003b, 13-48.

Weber, Gregor: Träume und Visionen im Alltag der römischen Kaiserzeit. Das Zeugnis der Inschriften und Papyri, in: Quaderni Catanesi n.s. 4-5, 2005-2006, 55-121.

Weber, Gregor: Die Alpträume der römischen Kaiser in Prinzipat und Spätantike. Definition - Begleitumstände - diskursive Kontexte, in: Jean-Marie Husser/Alice Mouton (Hg.): Le cauchemar dans les sociétés antiques, Paris 2011, 113-125.

White, Peter: Bookshops in the Literary Culture of Rome, in: William A. Johnson/ Holt N. Parker (Hg.), Ancient Literacies. The Culture of Reading in Greece and Rome, Oxford 2009, 268-287.

Winsbury, Rex: The Roman Book. Books, Publishing and Performance in Classical Rome, London 2009. 
Wiseman, Timothy P.: Augustus, Sulla and the Supernatural, in: Christopher Smith/ Anton Powell (Hg.), The Lost Memoirs of Augustus and the Development of Roman Autobiography, Swansea 2009, 111-123. 